\title{
Polysèmes
}

Revue d'études intertextuelles et intermédiales

\section{Tourner autour du vase chinois : poétique de l'immobilité vive chez T.S. Eliot}

Turning around the Chinese Jar: T.S. Eliot's Poetics of "Intense Immobility"

\section{Amélie Ducroux}

\section{(2) OpenEdition}

\section{Journals}

Édition électronique

URL : http://journals.openedition.org/polysemes/2286

DOI : $10.4000 /$ polysemes. 2286

ISSN : 2496-4212

Éditeur

SAIT

Référence électronique

Amélie Ducroux, «Tourner autour du vase chinois : poétique de l'immobilité vive chez T.S. Eliot», Polysèmes [En ligne], 18 | 2017, mis en ligne le 30 novembre 2017, consulté le 01 mai 2019. URL : http://journals.openedition.org/polysemes/2286 ; DOI : 10.4000/polysemes.2286

Ce document a été généré automatiquement le 1 mai 2019.

Polysèmes 


\title{
Tourner autour du vase chinois : poétique de l'immobilité vive chez T.S. Eliot
}

\author{
Turning around the Chinese Jar: T.S. Eliot's Poetics of "Intense Immobility"
}

\author{
Amélie Ducroux
}

1 Le moment apparaît comme un motif dans la poésie de T.S. Eliot. Le terme motif est employé ici au sens de thème qui s'imprime et se répète, sous forme de références sans explicitation particulière, comme dans ces vers de "The Love Song of $\mathrm{J}$. Alfred Prufrock »: "Should I, after tea and cakes and ices, / Have the strength to force the moment to its crisis?» (Eliot 5-6), et comme moteur de l'exploration poéticophilosophique des Four Quartets, comme dans ce passage de «Burnt Norton», premier des quatre quatuors :

But only in time can the moment in the rose-garden,

The moment in the arbour where the rain beat,

The moment in the draughty church at smokefall

Be remembered; involved with past and future.

Only through time time is conquered. (Eliot 180)

Le moment est aussi évoqué, indirectement, à travers un certain nombre d'images incluant «the still point of the turning world ». L'emploi du nom point plutôt que du nom moment permet au poète de spatialiser cette unité insaisissable qu'est le moment. En situant le moment dans l'espace, il devient possible, par exemple, d'employer des prépositions, "at the still point of the turning world», ou des adverbes: "there the dance is ", comme dans cet autre passage de «Burnt Norton » :

At the still point of the turning world. Neither flesh nor fleshless;

Neither from nor towards; at the still point, there the dance is.

But neither arrest nor movement. And do not call it fixity,

Where past and future are gathered. Neither movement from nor towards,

Neither ascent nor decline. Except for the point, the still point,

There would be no dance, and there is only the dance. 
I can only say, there we have been: but I cannot say where.

And I cannot say, how long, for that is to place it in time. (Eliot 179)

" And do not call it fixity » ("Et ne l'appelez pas fixité », ou « immobilité »), Eliot préciset-il. Cet avertissement semble témoigner d'une inquiétude face au risque que son exploration poétique soit réduite à des concepts figés par des termes tels fixity. C'est ce même passage que cite D.W. Harding lorsqu'il avance que ce poème, "Burnt Norton", "crée un nouveau concept ", selon un procédé qui consiste notamment à "mettre en avant et rejeter aussitôt ${ }^{1}$ des concepts figés, préexistants, concepts que l'on aurait été tenté d'assimiler au « concept » qui s'élabore sous nos yeux sur le mode poétique : «[...] for the purpose, essentially, of putting forward and immediately rejecting ready-made concepts that might have seemed to approximate to the concept he is creating" (Harding 109). Il dit par ailleurs du poème : « It makes no statement. It is no more "about" anything than an abstract term like "love" is about anything: it is a linguistic creation. And the creation of a new concept, with all the assimilation and communication of experience that that involves, is perhaps the greatest of linguistic achievements " (Harding 109). C'est précisément le rejet des binarités conceptuelles par la négation des substantifs ou prépositions qui les désignent ("neither arrest nor movement», «[n]either movement from nor towards») qui permet ici au poète d'explorer la complexité du moment autrement, sur un mode poétique qui pourrait prétendre ici sans doute au titre de méthode théorique visant à forger des concepts. L'emploi de la négation, «[n]either [...] nor ", la répétition de cette négation, permettent au poète d'exprimer la nature fluide et insaisissable du moment, l'impossibilité de le fixer, ou la nécessité pour le poète de ne pas le faire. À vouloir fixer le point, le poète risque en effet de se retrouver fort désappointé, car le point géométrique est toujours surreprésenté symboliquement, par une marque rendue visible, le point d'intersection de deux droites, quand lui-même ne l'est pas, puisqu'il n'a ni surface, ni longueur, ni largeur ni épaisseur. Sa représentation symbolique tend à fixer le point (entre deux droites) mais ne fait jamais que pointer le point comme concept théorique, à la fois présent et absent, visible et invisible.

Appréhender le moment, qui serait le pendant temporel du point spatial, apparaitt comme la tentative de le saisir en tentant de représenter une durée elle-même insécable et irreprésentable, obtenant ainsi, comme l'écrit Henri Bergson dans L'Évolution créatrice, " une imitation artificielle de la vie intérieure, un équivalent statique qui se prêtera mieux aux exigences de la logique et du langage, précisément parce qu'on en aura éliminé le temps réel. [...] notre durée n'est pas un instant qui remplace un instant [...] la durée est le progrès continu du passé qui ronge l'avenir et qui gonfle en avançant » (Bergson 3). Le moment ne relève que d'un pointage, d'une mise en relief mais il n'a pas d'existence en dehors de ce pointage :

Il est vrai que notre vie psychologique est pleine d'imprévu. Mille incidents surgissent, qui semblent trancher sur ce qui les précède, ne point se rattacher à ce qui les suit. Mais la discontinuité de leurs apparitions se détache sur la continuité d'un fond où ils se dessinent et auquel ils doivent les intervalles même qui les séparent: ce sont les coups de timbale qui éclatent de loin en loin dans la symphonie. Notre attention se fixe sur eux parce qu'ils l'intéressent davantage, mais chacun d'eux est porté par la masse fluide de notre existence psychologique toute entière. Chacun d'eux n'est que le point le mieux éclairé d'une zone mouvante qui comprend tout ce que nous sentons, pensons, voulons, tout ce que nous sommes enfin à un moment donné. C'est cette zone entière qui constitue, en réalité, notre état. Or, des états ainsi définis on peut dire qu'ils ne sont pas des éléments distincts. Ils se continuent les uns les autres en un écoulement sans fin. (Bergson 3) 
5 L'emploi de l'adverbe there dans le passage précédemment cité, (« at the still point [...] there the dance is ») pourrait suggérer qu'un lieu a été trouvé, le marqueur TH- pouvant pointer vers un lieu repéré à l'avance, ici peut-être seulement imaginé, rêvé, un lieu restant pourtant éloigné, «là-bas ». Ce pointage ne vaut qu'en tant que pointage infini puisque le lieu en question est dit ne pas être assignable en dehors de ce pointage : «I can only say, there we have been: but I cannot say where ». Ce « lieu » est toujours le lieu d'un renvoi, vers un avant, ou un après, un pointage en arrière ou en avant. " There » ne fait qu'annuler la stase qu'aurait pu suggérer la préposition $a t^{2}$. La « danse » est quant à elle mouvement, et non stase. Mais, plus que cela, cette «danse » qu'Eliot tente de décrire dans Four Quartets est le mouvement de ce qui ne peut rester en place, de ce qui oscille, vacille sans cesse, dans un mouvement perpétuel. Le poète dit des mots, dans la dernière section de «Burnt Norton », qu'ils ne peuvent "rester en place», ne peuvent "rester tranquilles » ou « immobiles » («[...] will not stay in place, / Will not stay still ») :

Words move, music moves

Only in time; but that which is only living

Can only die. Words, after speech, reach

Into the silence. Only by the form, the pattern,

Can words or music reach

The stillness, as a Chinese jar still

Moves perpetually in its stillness.

Not the stillness of the violin, while the note lasts,

Not that only, but the co-existence,

Or say that the end precedes the beginning,

And the end and the beginning were always there

Before the beginning and after the end.

And all is always now. Words strain,

Crack and sometimes break, under the burden,

Under the tension, slip, slide, perish

Decay with imprecision, will not stay in place,

Will not stay still. Shrieking voices

Scolding, mocking, or merely chattering,

Always assail them. The Word in the desert

Is most attacked by voices of temptation,

The crying shadow in the funeral dance,

The loud lament of the disconsolate chimera (Eliot 181-182)

La «forme» qui permet aux mots de s'inscrire en dépit de leur faiblesse, de leur vulnérabilité, de leur friabilité, est le poème lui-même. Mais cette « immobilité » n'est pas fixité. C'est une immobilité mouvante, une « immobilité vive ».

6 Si le vase chinois peut se mouvoir dans son immobilité, c'est parce que quelqu'un le regarde, l'appréhende, lui tourne autour, et, ce faisant, le met en mouvement. La même chose peut être dite du poème, car le poème imprimé, sans une paire d'yeux balayant sa surface, n'est qu'une apparition de marques immobiles. L'image du vase chinois semble être employée par Eliot pour suggérer à la fois la nécessité d'un agent qui lit et relie, le lecteur qui va connecter les signes entre eux, soulignant au passage l'impossibilité pour tout signe de faire sens en dehors du système qu'est le langage, et en dehors de cette unité complexe qu'est le poème, et la nature paradoxale du poème comme durée et moment, moment de suspension, ou élan (momentum).

7 Les mots prennent du temps. Il faut du temps pour les écrire, et du temps pour les lire. Pourtant, ce que vise Eliot est cette suspension du temps qui est centrale dans Four Quartets, ce "moment in and out of time» dont tout un chacun peut faire 
l'expérience, quand quelque chose est aperçu/perdu, quand quelque chose apparait sans pouvoir être saisi, moment qui, chez Eliot, ne peut se représenter que comme visée, ou pointage. C'est sur le mode poétique qu'Eliot appréhende de tels moments, même si la conscience du fait que toute tentative à cet égard ne peut mener qu'à l'échec trouve à s'exprimer à travers la voix de « East Coker » :

So here I am, in the middle way, having had twenty years -

Twenty years largely wasted, the years of l'entre-deux guerres -

Trying to use words, and every attempt

Is a wholly new start, and a different kind of failure

Because one has only learnt to get the better of words

For the thing one no longer has to say, or the way in which

One is no longer disposed to say it. And so each venture

Is a new beginning, a raid on the inarticulate

With shabby equipment always deteriorating

In the general mess of imprecision of feeling,

Undisciplined squads of emotion. And what there is to conquer

By strength and submission, has already been discovered

Once or twice, or several times, by men whom one cannot hope

To emulate - but there is no competition -

There is only the fight to recover what has been lost

And found and lost again: and now, under conditions

That seem unpropitious. But perhaps neither gain nor loss.

For us, there is only the trying. The rest is not our business. (Eliot 190)

8 Si le moment d'intensité ne peut être saisi à proprement parler, Eliot semble aussi vouloir dire qu'il ne doit pas être saisi, non pas tant par désir de préserver l'instant d'une inscription qui en altérerait l'expérience comme expérience vécue, mais par désir de se protéger de la potentielle intensité, pour ne pas dire violence du moment douloureusement vécu comme "toujours déjà » perdu. Dans "Burnt Norton ", Eliot relève l'adverbe there pour insister, peut-être, sur ce qui, dans l'expérience de l'instant, échappe toujours au sujet, est toujours déjà éloigné de lui :

I can only say, there we have been: but I cannot say where.

And I cannot say, how long, for that is to place it in time.

The inner freedom from the practical desire,

The release from action and suffering, release from the inner

And the outer compulsion, yet surrounded

By a grace of sense, a white light still and moving,

Erhebung without motion, concentration

Without elimination, both a new world

And the old made explicit, understood

In the completion of its partial ecstasy,

The resolution of its partial horror.

Yet the enchainment of past and future

Woven in the weakness of the changing body,

Protects mankind from heaven and damnation

Which flesh cannot endure. (Eliot 179)

Dire « there » revient à pointer en direction d'un moment et/ou d'un lieu de l'expérience déjà éloigné ("there we have been»), et sans limiter ni fixer les contours de cette expérience. L'expérience évoquée dans ce passage pourrait être d'ordre spirituel («the release from action and suffering, release from the inner / And the outer compulsion »), pourrait être comprise comme une forme de dépossession de soi, rappelant les pratiques bouddhistes. Pourtant «there ", « là-bas » il y a encore du mouvement, ou « là-bas, c'est encore du mouvement», «une lumière blanche immobile et mouvante»dont 
l'« intensité » se manifeste sous la forme d'une "concentration sans élimination » que nous pouvons comprendre comme une accumulation d'énergie. Comme le poète l'explicite, dans un des nombreux moments discursifs des Four Quartets, ce "moment ", cette révélation, n'équivaut pas à un changement radical menant à un «nouveau monde ", mais plutôt, « l'ancien rendu explicite »; l'« explicitation » elle-même ne vient pas du soulèvement d'un voile qui aurait caché quelque signification, mais d'une "concentration", d'une intensité faisant naître une nouvelle compréhension de l'«ancien monde». Il peut sembler paradoxal de relier "concentration» et " explicitation ", le premier mouvement suggérant une accumulation de matière pouvant elle-même entraîner une complexification empêchant l'appréhension directe de la chose, tandis qu' « explicitation » ou « explication » implique un déroulé du sens, ou, du moins, de différentes strates d'expérience ou de texte qui étaient jusqu'alors apparues comme un tout. Cette idée de concentration dans sa relation à l'image peut être reliée au fameux manifeste imagiste d'Ezra Pound, « A Few Don'ts by an Imagiste » :

An "Image" is that which presents an intellectual and emotional complex in an instant of time [...]

It is the presentation of such a "complex" instantaneously which gives that sense of sudden liberation; that sense of freedom from time limits and space limits; that sense of sudden growth, which we experience in the presence of the greatest works of art.

It is better to present one Image in a lifetime than to produce voluminous works. (Pound 1913, 200-201)

10 Pound insiste sur l'interdépendance de la complexité et de l'instantanéité ; il décrit également la sensation ressentie comme «la sensation d'une croissance soudaine » («a sense of sudden growth»), s'accompagnant d'une "sensation de liberté » et de «libération ». Il n'y a pas, pour reprendre le terme d'Eliot, d'«élimination», ou, pourrions-nous dire de réduction, mais une « croissance » potentiellement infinie qui ne connaît de limites ni de temps ni de lieu et permet au lecteur ou au spectateur de croître au-delà de ses propres limites, physiques mais aussi intellectuelles et émotionnelles. Cette " croissance », cette expansion au-delà de ses propres frontières, cette « libération ", sont des formes d'échappée du moi que l'instant, recomposé, représenté comme à la fois «dans le temps et en-dehors du temps » permet. L'on est tenté de dire que l'image, en particulier l'image «imagiste » selon Pound, très influencée par l'esthétique du haïku, nous permet de saisir quelque chose au vol. Ce qui reste non-dit, mais peut-être imperceptiblement suggéré dans la définition de Pound, est que cette «libération » a trait, en partie, au fait que l'image est vue et perdue simultanément, au même moment, que quelque chose nous échappe, dans le poème, dans le tableau, que quelque chose est vu dans son impossibilité même de se montrer. Cela n'est pas sans rappeler le punctum de Roland Barthes, qu'il décrit comme un « détail [qui] emporte toute [sa] lecture [...] une mutation vive de [son] intérêt, une fulguration ; [...] la marque de quelque chose [...] [qui] a fait tilt, [...] a provoqué en [lui] un petit ébranlement, un satori, le passage d'un vide " (Barthes 80-81). L'image est ce qui nous regarde mais ne peut être saisi dans sa totalité. Ce qui échappe est, de fait, central, aussi central que le vide du vase chinois d'Eliot est central. Cette image serait, à cet égard, l'image par excellence de l'image, à la fois du poème comme image, et de l'image dans le poème. Le lecteur «saisit " l'image du vase chinois "tournant perpétuellement dans son immobilité », la saisit en un instant, mais elle lui échappe aussi, en raison des ramifications intertextuelles de l'image, ici, par exemple, la possible référence au célèbre poème de John Keats « Ode on a Grecian Urn »" , ou de ses ramifications plus personnelles. Cette image poétique ne se contente pas de 
faire voir le vase, elle se fait vase, pour ainsi dire, en ce que le déchiffrement, la lecture de ses signes, met en acte le mouvement même qu'elle signifie, le mouvement du vase immobile qui se meut pourtant.

11 Ce qui est saisi dans/par l'image est comme un prélèvement opéré sur le sens infini, qui permet d'entrevoir ce sens sans jamais pouvoir l'embrasser complètement, ou, pour reprendre les termes de Jean-Luc Nancy dans Au fond des images : « Chaque image est un détourage fini du sens infini, lequel n'est avéré infini que par ce détourage, par le trait de la distinction. La surabondance des images dans la multiplicité et dans l'historicité des arts répond à l'inépuisable distinction. Mais chaque fois, en même temps, c'est la jouissance du sens, la secousse et le goût de sa tension: un peu de sens à l'état pur, infiniment ouvert ou infiniment perdu (comme on voudra dire) » (Nancy 30-31).

Le terme détourage suggère à la fois la séparation, la mise en valeur, la saillance de l'image, et sa relation avec un fond qui, parce qu'il a été supprimé, devient un fond ouvert sur l'infini, ou l'infini comme fond ouvert. La mise en valeur, l'insistance, la saillance de l'image est ainsi indissociable d'une ouverture sur cet infini sans fond. C'est pourquoi, lorsque Jean-Luc Nancy parle de l'« évidence » de l'image, l'on entend aussi l'opération par laquelle l'image, tout en s'inscrivant, en appelle à un vide, qu'il faudrait considérer comme caisse de résonance plutôt que comme vide négatif : «Claire et distincte, l'image est une évidence. Elle est l'évidence du distinct, sa distinction même. Il n'y a d'image que lorsqu'il y a cette évidence : sinon, il y a décoration ou illustration, c'est-à-dire soutien d'une signification. L'image doit toucher à la présence invisible du distinct, à la distinction de sa présence » (Nancy 30). Nancy insiste ici sur la nécessité pour l'image de faire sens sans se "[soutenir] d'une signification». L'image crée du sens, fait sens, rendant visible un invisible, un non-présent qui ne peut être situé cependant en-dehors de ce que produit l'image elle-même, rendant impossible toute assignation de l'image à ce qui serait son parfait envers, son prétendu, sous-entendu signifié.

Le vase chinois d'Eliot se fait image de l'image en ce qu'elle représente cet « arrachement de l'être à l'être » dont parle Nancy, "port[ant] en elle la marque de cet arrachement: son fond monstrueusement ouvert au fond d'elle, c'est-à-dire au revers sans fond de sa présentation (le dos aveugle du tableau) » (Nancy 51). Le vase chinois est, pouvons-nous penser, un vase sans fond. L'image échappe, aussi, en ce qu'elle ne devrait jamais être saisie complètement. Qu'est-ce qu'une image poétique parfaite, au juste, sachant que l'image est toujours déjà une figure de déplacement. La perfection de l'image réside-t-elle dans la perfection de l'illusion qu'elle tente de créer, une parfaite illusion de ressemblance qui serait jetée au visage du lecteur en un instant, le laissant sans voix ? Ou la comparaison figurative n'est-elle pas d'autant plus «parfaite » qu'elle laisse de l'espace entre son possible comparé et ce que suggère le phore, une faille de potentielle incertitude dans laquelle s'entrevoit, par la présence touchante de l'image, un absolu, ou une vérité en tant que présence absente, qui contribuera, sans doute, à produire l'émotion artistique. L'image « convient » précisément en ce qu'elle ne peut se rattacher à un objet, mais en ce qu'elle « se resserre sur soi » écrit Jean-Luc Nancy (Nancy 26). L'image, qu'elle soit métaphore, allégorie, comparaison, ne doit pas être ramenée en-deçà d'elle-même vers un quelconque référent, mais toujours être perçue comme pointant vers sa plus propre intimité, son cœur, qui est sans doute aussi son au-delà, son "évidence », son appel au sens absolu qui ne se forme comme appel qu'en vertu de son propre manque d'absolu ou de sa propre finitude : "Ainsi en va-t-il, selon une autre exemplarité, de ce qu'on nomme "l'image poétique". Cette dernière n'est pas la décoration fournie par un 
jeu d'analogie, de comparaison, d'allégorie, de métaphore ou de symbole. Ou bien, dans chacune de ces possibilités, elle est autre chose que le jeu plaisant d'un déplacement chiffré » (Nancy 28). Ces propos sur le « resserrement sur soi » de l'image rejoignent le commentaire de Barthes sur cette «immobilité vive» qui relie le haïku et la photographie : « liée à un détail (à un détonateur), une explosion fait une petite étoile à la vitre du texte ou de la photo : ni le haïku ni la Photo ne font "rêver" " (Barthes 81-82). La "petite étoile » marque la forme de l'insistance qui ne "peut que se répéter ", l'absence de "développement» de l'image. Y a-t-il pour autant contradiction à dire que l'image poétique ne renvoie qu'à elle-même en tant que détonation, et renvoie en même temps à un infini du sens ? Si le haïku et le punctum ne « font pas rêver ", ne renvoient pas au-delà d'eux-mêmes, ils n'en constituent sans doute pas moins, au même titre que l'image poétique frappante, un appel vers le sans-fond du langage, et peut-être, de la littérature, un fond infini en vertu du détourage que toute nouvelle image, mais aussi toute nouvelle production linguistique, et toute nouvelle œuvre, constitue toujours.

image poétique, aussi concise et percutante soit-elle, est toujours ramifiée, par ses évocations d'autres images, d'autres textes, en particulier dans la poésie d'Eliot, par la différance de l'écriture. La comparaison "[as] a Chinese jar moves perpetually in its stillness " provoque un impact, instantané, mais se marque aussi comme point de fuite, ou comme écart linguistique. Le nom jar, par exemple, fait aussi entendre le verbe jar, qui peut signifier, justement, « bouger dans une secousse » ou « être en décalage ».

15 Le fait que le " point fixe " doive être mouvant, comme Eliot le souligne sur des modes divers et grâce à différentes images tout au long des Four Quartets est crucial, parce que le mouvement de l'immobilité, cette impossible possibilité, est ce qui garantit la possibilité de l'émotion née de l'espacement. Sans l'espacement, sans une forme de dis-location, parfois si infime qu'elle échappe presque à notre attention, il n'y aurait pas de saisie dans la perte, ou de perte dans la saisie, cet essentiel mouvement de l'émotion.

16 Eliot emploie le nom dance pour exprimer ce mouvement immobile. La danse est mouvement qui ne peut être fixé. L'on peut regarder danser un danseur, l'on peut enregistrer une performance, toujours en mouvement ; si l'on photographie un danseur, cela devient l'image de quelqu'un pris dans l'acte de danser. Et le seul moyen de retrouver ce mouvement perdu sera de trouver, peut-être, le punctum dans notre image du danseur ou la trace, peut-être, de son "avoir été ", pour faire référence à Barthes regardant la photo de sa mère enfant, ou de son « avoir dansé ». Le danseur, dansant, reste le danseur, comme le vase chinois, tournant, reste le vase chinois. L'immobilité, par conséquent, est sans doute ce qui s'offre à la vue, comme semblant d'intégrité, de présence; mais le mouvement est ce qui déplace non seulement le sujet ou l'objet qui bouge, mais aussi celui qui l'appréhende, le lecteur, le spectateur déplacé, retourné, bouleversé, é-mu.

Le fait qu'Eliot emploie l'image du vase, un objet ouvert, n'est pas anodin. Son ouverture même est le point central, pour ainsi dire, en gardant à l'esprit que le centre comme point n'est jamais qu'une entité s'évanouissant à l'horizon, il ne peut être touché et son approche ne mène qu'à un déplacement ou une régression infinis. Ainsi, le vide du vase est, littéralement et figurativement, central. Il permet au vase de tourner sur lui-même, au spectateur de lui tourner autour, tout en entourant un vide intérieur et sans fond, qui toujours nous échappe. Le moment apparait comme le cœur de l'écriture poétique d'Eliot, point de fuite qui ouvre sur l'abîme et point vital sans lequel la «danse " poétique ne saurait avoir lieu. En appréhendant le moment, en tournant autour du point, le poème ne cesse de rechercher son centre et, par cette recherche même, l'évite et l'évide en même 
temps. Le mouvement de saisie/dessaisissement constitue la dynamique de son écriture et peut-être aussi son seul « objet », ou objectif. Cet objectif, à la fois motif et objectif, que le moment représenterait, ne serait rien d'autre que ce qui échappe toujours à l'écriture. En appréhendant le moment, le poème inscrit ainsi son brillant échec. Cette vaine tentative qu'est le poème se fait cependant trace d'une visée du moment, d'un désir de saisie ou de ressaisie, offrant au lecteur la possibilité de faire l'expérience de cette appréhension de ce qui, précisément, ne peut être « saisi » que dans le mouvement qui ne fait que tenter de s'en emparer. Le poème tout entier se fait l'image de ce mouvement, il le fixe tout en préservant sa vitalité dans l'espoir d'une relance par la lecture. L'image du "vase chinois» de "Burnt Norton» exprime cette immobilité vive, cette immobilité tournante qu'est aussi le poème, l'objet (vase et poème) étant lui-même formé autour d'un vide, voire d'une absence, qui le constitue et qu'il enveloppe. Si l'on voit ce vase comme image du poème, cela implique que, quelque image que l'on saisisse et quels qu'en soient les effets sur le lecteur, ces derniers se soutiendront toujours aussi de ce qui lui aura essentiellement échappé, marquant sa place dans le règne du signifiant et de la représentation, et que l'on pourra situer, sans doute, sur l'envers du poème.

Pour conclure, je citerai à nouveau Jean-Luc Nancy : «L'art n'est pas ce simulacre ou cette forme apotropaïque (la "vérité-Gorgone » selon Nietzsche, la "pulsion aveugle » selon Freud). Il est le savoir exact de ceci qu'il n'y a rien à révéler, pas même un abîme, et que le sans-fond n'est pas le gouffre d'une conflagration, mais l'imminence infiniment suspendue sur soi» (Nancy 56). Cette «imminence infiniment suspendue sur soi» pourrait être l'immobilité vive créée par l'œuvre, prise entre la fixité de la représentation (picturale, linguistique), qui serait ici «suspension» et le mouvement, intense, insoutenable, de l'« imminence ", que l'on pourrait rapprocher du momentum, du moment vif. Ainsi, lorsque la voix de "The Love Song of J. Alfred Prufrock" se demande avec inquiétude : "Should I, after tea and cakes and ices, / Have the strength to force the moment to its crisis?", ne se fait-elle pas le relais du poète se demandant s'il aura la force, le courage, de forcer le moment jusqu'à sa crise, autrement dit, peut-être, d'écrire le moment, opération qui n'est de fait, qu'un "forçage », une mise en évidence de ce qui se saisit en tant que perte dans le moment.

\section{BIBLIOGRAPHIE}

Barthes, Roland. La Chambre claire. Note sur la photographie. Paris : Cahiers du cinéma/Gallimard/ Éditions du Seuil, 1980.

Bergson, Henri. L'Évolution créatrice (1907). Paris : PUF, « Quadrige », 2013.

Eliot, T.S. Collected Poems 1909-1962. London: Faber and Faber, 1974.

Harding, D.W. “T.S. Eliot, 1925-1935”. T.S. Eliot. A Collection of Critical Essays. Hugh Kenner (ed.). Englewood Cliffs: Prentice-Hall, Inc., 1962.

Keats, John. The Poetical Works of John Keats. H.W. Garrod (ed.). London: Oxford UP, 1956.

Nancy, Jean-Luc. Au fond des images. Paris : Galilée, 2003. 
Pound, Ezra. “A Few Don’ts by an Imagiste”. Poetry 1.6 (March 1913): 200-206.

\section{NOTES}

1. Je traduis.

2. La préposition at peut aussi renvoyer au fait de se diriger vers, d'entrer en contact avec, exprimant alors un mouvement d'approche.

3. «O Attic shape! Fair attitude! with brede / Of marble men and maidens overwrought, / With forest branches and the trodden weed; / Thou, silent form, dost tease us out of thought / As doth eternity: Cold Pastoral! / When old age shall this generation waste, / Thou shalt remain, in midst of other woe / Than ours, a friend to man, to whom thou say'st, / "Beauty is truth, truth beauty, -that is all / Ye know on earth, and all ye need to know" " (John Keats, "Ode on a Grecian Urn » [1820], The Poetical Works of John Keats, London: Oxford UP, 1956, 210).

\section{RÉSUMÉS}

Le moment, motif central dans la poésie de T.S. Eliot, ne peut être saisi, ne peut être que pointé du doigt ou représenté symboliquement, car à qui veut le saisir, il n'offre que le constat d'une perte. Ce moment que la voix de «The Love Song of J. Alfred Prufrock » évite et appelle tout à la fois, n'est-il pas, au-delà d'une «question bouleversante " que nulle interprétation ne saura mettre au jour, au-delà d'un moment d'expérience dont l'intensité pourrait ébranler le sujet jusqu'à sa perte, la révélation du dessaisissement qui accompagne toute tentative d'attraper le moment au vol? Comment la poésie d'Eliot parvient-elle à exprimer ce mouvement contradictoire? Dans Four Quartets, Eliot a appréhendé le moment en une variation poéticophilosophique autour de ce motif, motif qui prend la forme d'images, dont le «still point of the turning world » reste la plus frappante. Le moment est appréhendé non plus en tant qu' « unité » temporelle désirable et insaisissable, mais en tant que "point " inassignable, à la fois fixe et mouvant, «du monde qui tourne». L'image du «vase chinois» («Chinese jar») dans «Burnt Norton » semble exprimer cette "immobilité vive», cette immobilité tournante qu'est aussi le poème. Cette image sera le point d'appui de cette appréhension de l'« immobilité vive » au cœur de la poésie d'Eliot, une poésie qui, dans Four Quartets notamment, devient lieu d'élaboration de concepts, lieu autre mais non moins légitime ni non moins productif que celui du discours.

The moment, a central motif in T.S. Eliot's poetry, cannot be seized but only pointed out or represented symbolically. The very attempt to grasp it leaves one with the bitter realization that it is already behind. This moment the speaker of "The Love Song of J. Alfred Prufrock" avoids and anticipates at the same time should not be reduced to an "overwhelming question" that no interpretation will ever fathom, nor to the moment of an experience whose intensity may threaten the subject; it may also reveal that any attempt to catch it implies to let it go. How is this contradictory movement expressed in Eliot's poetry? In Four Quartets, Eliot deals with the moment not as a temporal "unit"-both desirable and ungraspable-but as the unassignable, undecidable point-still and moving-"of the turning world". The image of the "Chinese jar" in "Burnt Norton" could be the "objective correlative" of this "intense immobility", this "turning immobility", which may also refer to the poem itself. In this approach to the "intense 
immobility" in Eliot's poetry, partly relying on this image, I would like to suggest also, as some critics have already pointed out, that the poem becomes the soil from which concepts grow, different from the soil of discourse, but not less legitimate nor less productive.

INDEX

Mots-clés : moment, image, concept

Keywords : moment, image, concept oeuvrecitee Four Quartets

\section{AUTEURS}

\section{AMÉLIE DUCROUX}

Amélie Ducroux est maître de conférences à l'Université Lumière - Lyon 2 où elle enseigne la littérature américaine. Ses travaux de recherche portent principalement sur la poésie et le modernisme. Elle a publié en 2014 La relation et l'absolu. Lectures de la poésie de T.S. Eliot aux Presses de l'Université Paris-Sorbonne (collection Americana), ouvrage issu de sa thèse. 